\section{Military Technical College Kobry El-kobbah, Cairo, Egypt}

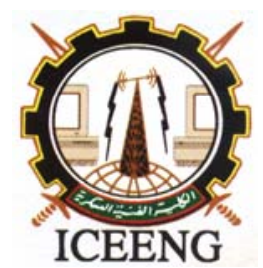

\section{$5^{\text {th }}$ International Conference on Electrical Engineering ICEENG 2006}

\title{
Performance Analysis of Hybrid DS/SFH System Over AWGN Channel
}

\author{
Col. Dr.: Abdelmoneim M. Fouda, Lt. Col. Dr. Ezz E. Farouk, Eng. G. H. Alshamari
}

\begin{abstract}
In recent years, the trend in the spread spectrum communication systems is to use the combined hybrid techniques. The use of a hybrid systems attempt to capitalize upon the advantages of a particular method while avoiding the disadvantages. Many different hybrid combinations are possible; those are: DS/FH hybrid system, DS/time hopping hybrid system, FH/time hopping hybrid system, and DS/FH/time hopping hybrid system.

In this paper, we will be concern on the DS/FH hybrid system that can combine the advantages of both DS and FH while avoiding some of their disadvantages. This hybrid system can combine the anti-multipath effectiveness of DS system with good anti-jamming and good anti-far problem features of FH systems. Moreover, the hybrid system has shorter signature sequences and hopping patterns, thus reducing the overall acquisition time. While a disadvantage of hybrid systems is the increased complexity of their transmitter and receiver.

The paper presents general closed form of the system BER formula which will be derived assuming non-coherent hybrid DS/SFH-SSMA over AWGN channel and employing MFSK. Moreover, the analysis is extended to include the derivation of the closed form of BER for both the pure FH and pure DS SSMA. Then, the hybrid DS/SFH system performance measures is compared with the performance of both the pure DS-SSMA and the pure FHSSMA system subjected to the same channel conditions.
\end{abstract}

\section{KEYWORDS:}

CDMA, spread spectrum, DS-SSMA, FH-SSMA.

\section{INTRODUCATION}

In the field of communication, it is noted that tremendous increase of the number of users sharing the same communication channels may cause a serious problem and leads to the degradation of the overall system performance. Spread spectrum multiple access (SSMA) is one of the multiple-access capabilities which has proven great advantages to overcome such problem. In addition to the SSMA techniques, it provides other desirable qualities including considerable effectiveness in combating various types of intentional interference (jamming) or non-intentional interference.

SSMA is by far the most popular form of code division multiple access (CDMA). The most commonly used forms of SSMA are direct-sequence SSMA (DS/SSMA), in which a high-rate code (termed a signature sequence) is used together with the data signal to phase modulate the carrier signal. On the other hand; frequency-hopped SSMA (FH/SSMA), in which a frequency-hopping pattern (high-rate code) is used to control a frequency-synthesized carrier signal onto which data are frequency modulated. Nowadays, the new trend is the hybrid 
SSMA. A disadvantage of the hybrid systems is the increased complexity of their transmitters and receivers.

From the literature survey it is noted that many researches in the past few years have been investigated the multiple-access capability of DS/SS (e.g., [1]-[2]) and FH/SS (e.g., [3]-[4]) systems have been thoroughly also investigated, hybrid DSFH/SSMA systems have not received sufficient attention.

Throughout this paper we are concerning on the performance of hybrid DS/SFH -SSMA employing the M-ary frequency shift keying (MFSK) systems; where during transmission part the original data is first DS/SS modulated and then frequency-hopped according to a specific frequency-hopping pattern. The considered system is operating through additive white Gaussian noise (AWGN) channels. The performance measure is the bit error rate (BER). Besides, the multiple-access capability, or the number of users that the system can deal with will be taken as measurement parameters.

The paper is organized as follows: in section II, the system model of the Hybrid DS/SFHMFSK assuming AWGN channel is introduced. In section III, we introduce to the analysis of the hybrid DS/SFH over the AWGN channel. In section V, numerical results are presented, and then the final conclusion about the paper is provided.

\section{System Model}

In the system model, we will find a complete simplified transmitter and receiver block diagram with waveforms that representing the different stages of such hybrid DS-SFH under considerations. The block diagram of the complete hybrid DS/SFH operating through AWGN channel is shown in Fig. 1. Our model for the hybrid DS/SFH-MFSK SSMA system combines features of both slow-frequency-hopping SSMA model, and the direct-sequence SSMA model, assuming $\mathrm{N}$ code pulses in each data pulses.

From Fig. 1, the waveform at the output of the modulator can be represented as [1]:

$$
\mathrm{C}_{\mathrm{k}}(\mathrm{t})=2 \sqrt{2 P} \quad \mathrm{a}_{\mathrm{k}}(\mathrm{t}) \cdot \mathrm{b}_{\mathrm{k}}(\mathrm{t}) \cos \left(2 \pi \mathrm{f}_{\mathrm{c}} \mathrm{t}+\theta_{\mathrm{k}}\right)
$$

Where $P$ is the power in each of the $K$ transmitted signals, $f_{c}$ is the carrier frequency, and $\theta_{k}$ is the phase angle introduced by $\mathrm{k}^{\text {th }}$ modulator spreader. From the figure shown, the DS/SS signal $C_{k}(t)$ is then frequency-hopped according to the $k^{\text {th }}$ hopping pattern $f_{k}(t)$ which is derived from random pattern sequence. The transmitted signal is given as:

$$
\mathrm{S}_{\mathrm{k}}(\mathrm{t})=\sqrt{2 P} b_{\mathrm{k}}(\mathrm{t}) \mathrm{a}_{\mathrm{k}}(\mathrm{t}) \cos \left(2 \pi\left[\mathrm{f}_{\mathrm{c}}+\mathrm{f}_{\mathrm{k}}(\mathrm{t})\right] \mathrm{t}+\theta_{\mathrm{k}}+\mathrm{u}_{\mathrm{k}}(\mathrm{t})\right)
$$

Where $P$ is the signal power, $f_{c}$ is the center frequency, while $f_{k}(t)$ is the hopping frequency, $\theta_{\mathrm{k}}$ is the random phase introduced by the $\mathrm{k}^{\text {th }}$ modulator spreader. Also $\mathrm{u}_{\mathrm{k}}(\mathrm{t})$ is the random phase introduced by the $\mathrm{k}^{\text {th }}$ user frequency hopper, $\mathrm{b}_{\mathrm{k}}(\mathrm{t})$ is the data signal that has the rectangular pulse shape of duration $\mathrm{T}_{\mathrm{b}}$ we assume that there are $\mathrm{N}_{\mathrm{b}}$ bits per hop; $\mathrm{a}_{\mathrm{k}}(\mathrm{t})$ is the spreading signal with chip duration time $\mathrm{T}_{\mathrm{c}}$.

During reception, the reverse process is performed. Thus, the first step is the frequency dehopping then followed by despreading and detection part for outputting data that must be different from the original transmitted data due to the effect of the AWGN and the multiple access inference. The received signal, $r(t)$ is given by:

$$
\begin{aligned}
\mathrm{r}_{\mathrm{k}}(\mathrm{t}) & =\sum_{k=1}^{K} \mathrm{~S}_{\mathrm{k}}\left(\mathrm{t}-\tau_{\mathrm{k}}\right)+\mathrm{n}(\mathrm{t}) \\
& =2 \sqrt{2 P} \mathrm{~b}_{\mathrm{k}}\left(\mathrm{t}-\tau_{\mathrm{k}}\right) \mathrm{a}_{\mathrm{k}}\left(\mathrm{t}-\tau_{\mathrm{k}}\right) \cos \left(2 \pi\left[\mathrm{f}_{\mathrm{c}}+\mathrm{f}_{\mathrm{k}}\left(\mathrm{t}-\tau_{\mathrm{k}}\right)\right]\left(\mathrm{t}-\tau_{\mathrm{k}}\right)+\theta_{\mathrm{k}}+\mathrm{u}_{\mathrm{k}}\left(\mathrm{t}-\tau_{\mathrm{k}}\right)\right)+\mathrm{n}(\mathrm{t})
\end{aligned}
$$


Where $n(t)$ is the AWGN with power spectral density No/2, $\tau_{k}$ is the time delay parameters, and $\mathrm{K}$ is the number of active users.

\section{Performance of Hybrid DS/SFH over AWGN channel}

Two different techniques are developed for the evaluation of the error Probability for the hybrid DS/SFH system performance under the effect of the AWGN mentioned as [5]:

- The characteristic-function method, which is based on the integration of the characteristic function of the multiple-access interference

- A technique based on evaluating the conditional error probability given the number of hits from other users and then averaging over the distribution of the hits.

Throughout this work, the evaluation method is based on the second technique. We consider the effect of the AWGN and the system employs the MFSK modulation technique. The probability of error of the AWGN channel with MFSK modulation can be expressed as [6]:

$$
p_{\text {AWGN }}=\frac{2^{b-1}}{2^{b}-1} \cdot \sum_{n=1}^{M-1}\left(\begin{array}{l}
M-1 \\
n
\end{array}\right) \frac{(-1)^{n+1}}{n+1} \cdot \exp \left\{\frac{-n}{(n+1)} \cdot \frac{E_{b}}{N_{o}} \cdot b\right\}
$$

where $E_{b} / N_{o}$ is the signal to noise ratio of the system which represents the ratio between the bit energy $E_{b}$ to the thermal noise density $N_{o}$, while $b$ is the number of bits in the information, and $\mathrm{M}$ is the order of the MFSK modulation.

Firstly, we will start the analysis with non-coherent asynchronous hybrid DS/SFH SSMA system with random signature sequences and hopping patterns independent of $i$. The general error probability BER of such system is given as [5]:

$$
\mathrm{P}_{\mathrm{hyp}}=\sum_{k_{f}}^{K-1} \sum_{k_{p}}^{K-1-k_{f}} p_{h}\left(k_{f}, k_{p}\right) p_{e}\left(k_{f}, k_{p}\right)
$$

where

- $\quad p_{e}\left(k_{f}, k_{p}\right)$ denotes the conditional error probability of the system given that $\mathrm{k}_{\mathrm{f}}$ full hits and $\mathrm{k}_{\mathrm{p}}$ partial hits occurred,

- $\quad p_{h}\left(k_{f}, k_{p}\right)$ denotes the probability of the occurrence of $\mathrm{k}_{\mathrm{f}}$ full hits and kp partial hits from the other K-1 users. This can be expressed as:

$$
p_{h}\left(k_{f}, k_{p}\right)=\left(\begin{array}{l}
K-1 \\
k_{f}
\end{array}\right)\left(\begin{array}{l}
K-1-k_{f} \\
k_{p}
\end{array}\right) p_{f}^{k_{f}} p_{p}^{k p}\left(1-p_{f}-p_{p}\right)^{K-1-k_{f}-k_{p}}
$$

where

- the full hits $\mathrm{k}_{\mathrm{f}}$ and partial hits $\mathrm{k}_{\mathrm{p}}$ with limited values given as: $0 \leq \mathrm{k}_{\mathrm{f}}<\mathrm{K}$ and $\mathrm{k}_{\mathrm{f}} \leq \mathrm{k}_{\mathrm{p}}<\mathrm{K}-1$

- $\quad p_{p}$ is the partial hits probability from other users, given as $P_{p}=2 /\left(N_{b} . q\right)$

- $p_{f}$ is the full hits probability from other given as : $p_{f}=\left(1-1 / N_{b}\right) / q$ 
All the above expressions are some of the parameters constituting the BER general form. Now to complete the analysis and to get the main target of obtaining a general closed form expression for the hybrid DS/SFH employing MFSK through AWGN channel ( with defined parameters; and that can be evaluated by a simulation software), we must get an expression of $p_{e}\left(k_{f}, k_{p}\right)$ which represent the conditional error probability of the system given that $\mathrm{k}_{\mathrm{f}}$ full hits and $\mathrm{k}_{\mathrm{p}}$ partial hits occurred, the conditional probability is:

$$
p_{e}\left(k_{f}, k_{p}\right)=\sum_{m=1}^{M-1}\left(\begin{array}{l}
M-1 \\
m
\end{array}\right) \frac{(-1)^{m+1}}{m+1} \cdot \exp \left\{\frac{-m}{2(m+1)}\left[\left(\frac{2 E_{b} \log _{2} M}{N_{0}}\right)^{-1}+\frac{\left(k_{f}+0.5 k_{p}\right)}{3 M \cdot N \log _{2} M}\right]^{-1}\right\}
$$

It is clear now that all parameters of the general form are clarified and identified. By using the different expressions of $p_{e}\left(k_{f}, k_{p}\right), p_{h}\left(k_{f}, k_{p}\right), p_{f}$, and $p_{p}$ and substitute into the general form of the BER equation $P_{\text {hyp }}$ then we get the final closed form of the non-coherent hybrid DS/SFH SSMA asynchronous system employing MFSK and operating through AWGN channel can be expressed as:

$$
\begin{gathered}
P_{h y p}=\sum_{k_{f}}^{K-1} \sum_{k_{p}}^{K-1-k_{f}} \sum_{m=1}^{M-1}\left(\begin{array}{l}
M-1 \\
m
\end{array}\right) \frac{(-1)^{m+1}}{m+1} \cdot \exp \left\{\frac{-m}{2(m+1)}\left[\left(\frac{2 E_{b} \log _{2} M}{N_{0}}\right)^{-1}+\frac{\left(k_{f}+0.5 k_{p}\right)}{3 M \cdot N \log _{2} M}\right]^{-1}\right\} \\
\cdot\left(\begin{array}{l}
K-1 \\
k_{f}
\end{array}\right)\left(\begin{array}{l}
K-1-k_{f} \\
k_{p}
\end{array}\right) p_{f}^{k_{f}} p_{p}^{k p}\left(1-p_{f}-p_{p}\right)^{K-1-k_{f}-k_{p}}
\end{gathered}
$$

Then after introducing some re-arrangement process and substitute with $\mathrm{P}_{\mathrm{f}}$ and $\mathrm{P}_{\mathrm{p}}$ then we modify the above equation and we get :

$$
\begin{aligned}
& P_{\text {hyp }}=\sum_{k_{f}}^{K-1} \sum_{k_{p}}^{K-1-k_{f}} \sum_{m=1}^{M-1}\left(\begin{array}{l}
M-1 \\
m
\end{array}\right)\left(\begin{array}{l}
K-1 \\
k_{f}
\end{array}\right)\left(\begin{array}{l}
K-1-k_{f} \\
k_{p}
\end{array}\right) \frac{(-1)^{m+1}}{m+1} \cdot\left(\frac{2}{N_{b} q}\right)^{k_{p}}\left(\frac{1-N_{b}^{-1}}{q}\right)^{k f} \\
& . \exp \left\{\frac{-m}{2(m+1)}\left[\left(\frac{2 E_{b} \log _{2} M}{N_{0}}\right)^{-1}+\frac{\left(k_{f}+0.5 k_{p}\right)}{3 M \cdot N \log _{2} M}\right]^{-1}\right\}\left(1-\left(\frac{2}{N_{b} q}\right)-\left(\frac{1-N_{b}^{-1}}{q}\right)\right)^{K-1-k_{f}-k_{p}}
\end{aligned}
$$

The above derived equation is considered as the general case of the SSMA, it represents the final closed form of hybrid DS/SFH system over the AWGN channel including the effect of the number of the active users $\mathrm{K}$ that utilizing and sharing the channel. Now we develop to conclude the two special cases derived from the above equation by controlling the value of the processing gain of the DS-SSMA individually $\mathrm{N}$, and the processing gain of the FH-SSMA individually q. Now we extend the analysis by putting $\mathrm{N}=1$ in the above equation; then we can get the final closed form of the non-coherent pure SFH SSMA system employing MFSK and operating through AWGN channel we get :

$$
\begin{aligned}
& P_{F H}=\sum_{k_{f}}^{K-1} \sum_{k_{p}}^{K-1-k_{f}} \sum_{m=1}^{M-1}\left(\begin{array}{l}
M-1 \\
m
\end{array}\right)\left(\begin{array}{l}
K-1 \\
k_{f}
\end{array}\right)\left(\begin{array}{l}
K-1-k_{f} \\
k_{p}
\end{array}\right) \frac{(-1)^{m+1}}{m+1} \cdot\left(\frac{2}{N_{b} q}\right)^{k_{p}}\left(\frac{1-N_{b}^{-1}}{q}\right)^{k f} \\
& . \exp \left\{\frac{-m}{2(m+1)}\left[\left(\frac{2 E_{b} \log _{2} M}{N_{0}}\right)^{-1}+\frac{\left(k_{f}+0.5 k_{p}\right)}{3 M \cdot \log _{2} M}\right]^{-1}\right\}\left(1-\left(\frac{2}{N_{b} q}\right)-\left(\frac{1-N_{b}^{-1}}{q}\right)\right)^{K-1-k_{f}-k_{p}}
\end{aligned}
$$


Also, when we put $\mathrm{q}=1$ in the above equation, we can get the final closed form of the pure DS-SSMA system employing MFSK and operating through AWGN channel as:

$$
\begin{aligned}
& P_{D S}=\sum_{k_{f}}^{K-1} \sum_{k_{p}}^{K-1-k_{f}} \sum_{m=1}^{M-1}\left(\begin{array}{l}
M-1 \\
m
\end{array}\right)\left(\begin{array}{l}
K-1 \\
k_{f}
\end{array}\right)\left(\begin{array}{l}
K-1-k_{f} \\
k_{p}
\end{array}\right) \frac{(-1)^{m+1}}{m+1} \cdot\left(\frac{2}{N_{b}}\right)^{k_{p}}\left(1-N_{b}^{-1}\right)^{k f} \\
& \cdot \exp \left\{\frac{-m}{2(m+1)}\left[\left(\frac{2 E_{b} \log _{2} M}{N_{0}}\right)^{-1}+\frac{\left(k_{f}+0.5 k_{p}\right)}{3 M \cdot N \cdot \log _{2} M}\right]^{-1}\right\}\left(1-\left(\frac{2}{N_{b}}\right)-\left(1-N_{b}^{-1}\right)\right)^{K-1-k_{f}-k_{p}}
\end{aligned}
$$

\section{Numerical Results}

We use the MATLAB software package to verify the equation of the AWGN channel without hybrid system, and the equation of the hybrid DS/SFH-MFSK operating through AWGN channel. A comparison between the system performance of the different system including the hybrid DS/SFH, the pure FH and the pure DS SSMA was analyzed under the same operating conditions. Fig.2 represents the performance of AWGN channel employing MFSK and its BER. The performance is achieved at different values of $M=4,8$, and 16 . It is found that as the modulation order increase, the system performance is better due to the increased number of bits per information symbol transmitted $b=\log _{2}(M)$, but the system complexity and cost is also increased as $\mathrm{M}$ increases.

The verification of the performance in that case of the hybrid system is shown in Fig.3 then it is compared in the same plot with the BER of AWGN that previously described. The scenario of calculations are achieved assuming that $K=1$, the number of frequency hops $q=100$, the number of chips per bits $N=31$. The number of bits per hops $\mathrm{N}_{b}=10$ bits/hop, the $\mathrm{SNR}$ is ranged from 0 to $12 \mathrm{~dB}$. It is noted from the plot that the system performance is affected slightly with the hybrid DS/SFH in the case of AWGN channel, the little decrease in the BER is due to spreading the signal power over wide band thus reduce the over all effects of the signal part compared to the noise part, and at high SNR they are approximately the same.

Now, we are going to study the system capacity by plotting BER versus the number of active users $K$ at $M=4$. The computational analysis is introduced at different values of $S N R=10 \mathrm{~dB}$, $12 \mathrm{~dB}$, and $14 \mathrm{~dB}$. From Fig.4, it is noted that for the same number of users the system BER is greatly enhanced with the increase of SNR.

For the purpose of comparison, we fix the $\mathrm{SNR}=10 \mathrm{~dB}$, the system capacity of the Hybrid DS/SFH-MFSK with $\mathrm{N}=31, \mathrm{q}=100$, while the pure FH-SSMA with $\mathrm{q}=100, \mathrm{~N}=1$, then the pure DS-SSMA with $\mathrm{N}=31, \mathrm{q}=1$. It is found from Fig.5 that the hybrid DS/SFH-SSMA outperforms the pure FH, and then followed by the DS-SSMA.

\section{Conclusion}

We have presented the performance analysis of the hybrid DS/SFH-SSMA technique under the effect of the AWGN channel conditions. A closed form expression that represent the BER of the hybrid system is derived as well as the closed form BER for the pure FH and DS multiple access techniques. It is found that the hybrid system outperforms the other spread spectrum techniques including the FH and the DS spread spectrum. Also, as the SNR level increases, the number of users utilizing the system also increases. 


\section{References}

[1] L. L. Yang, Hanzo L. "performance of generalized multicarrier DS-CDMA over nakagami-m fading channel ", IEEE Trans. On communication Vol. 50, No. 6 June 2002.

[2] Marvin K-Simon, Jim K-Omura, Robert A Scholtz and Barry K. Levitt, " Spread spectrum communications handbook," McGraw Hill Inc., 1994.

[3] Usa Svasti Xuto, Qiang Wang and Vijay K. Bhargava "Capacity of an FH-SSMA system in different fading environments ", IEEE Trans. On Vehicular Technology Vol. VT-47, No. 1 February 1998.

[4] Kah C. The, and Kwok H. Li, "Error Probabilities of an FFH/BFSK self normalizing receiver in a Rician fading channel with multitone jamming", IEEE Trans. On Comm., Vol 48, No. 2 February 2000.

[5] Evaggelos A. Geraniotis," Noncoherent hybrid DS-SFH spread spectrum multiple access communications", IEEE trans. Comm. Vol. Com-34, No. 9, September 1986.

[6] John G. Proakis "Digital communication", McGraw Hill , 2000. 


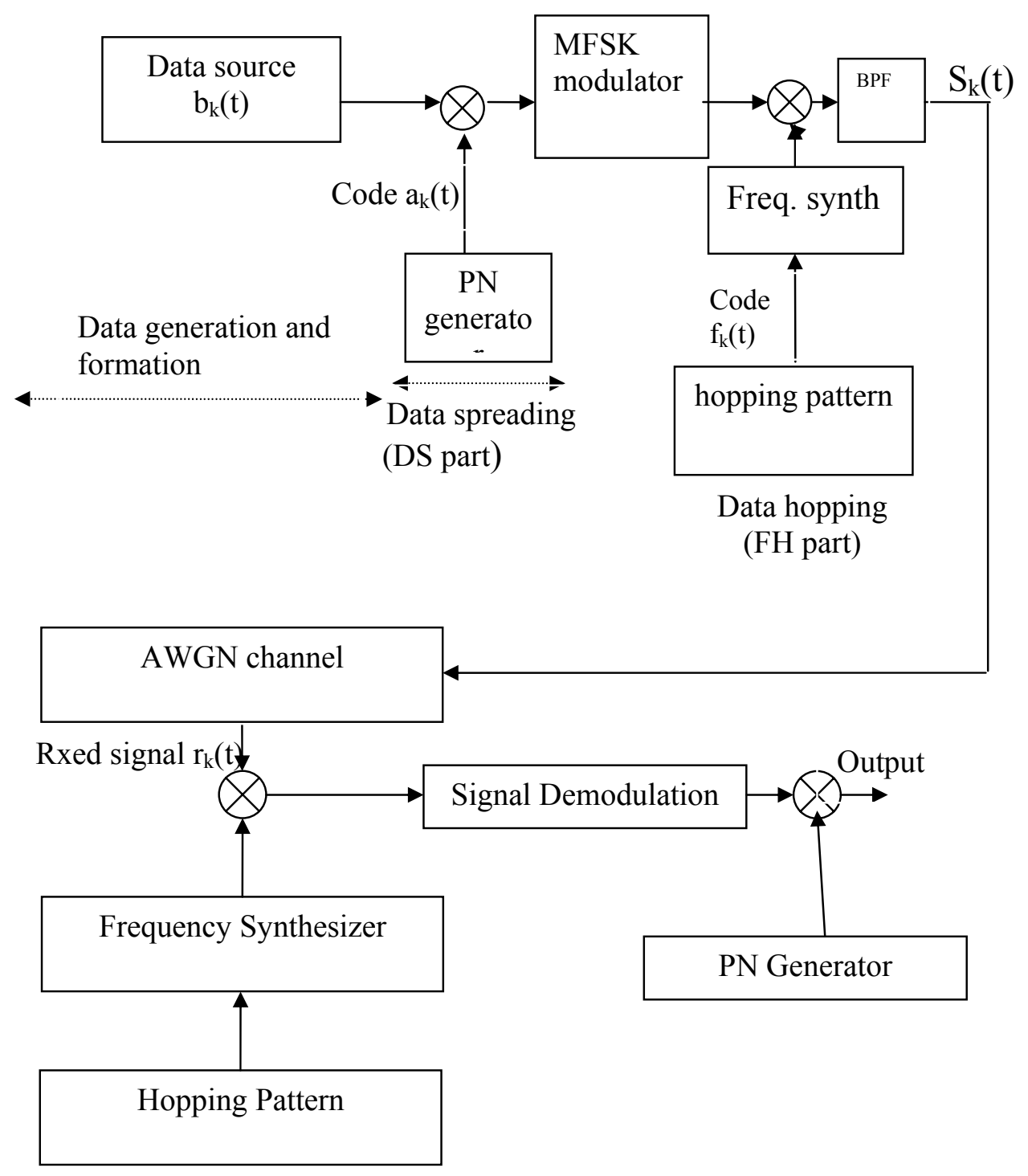

Fig. 1 General block diagram of the hybrid DS/SHF operating through AWGN Channel 


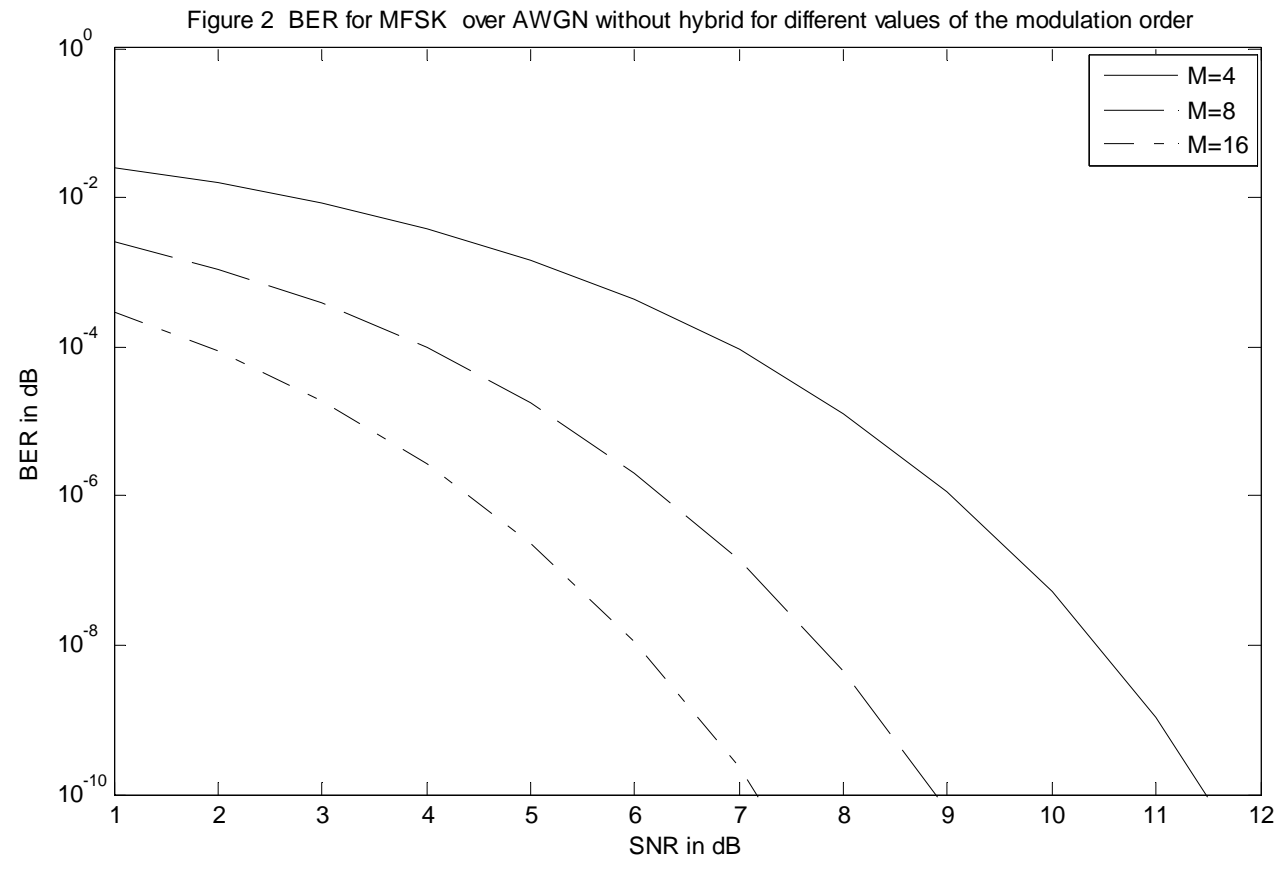

Fig. 2 BER for MFSK over AWGN without hybrid for different values of modulation order

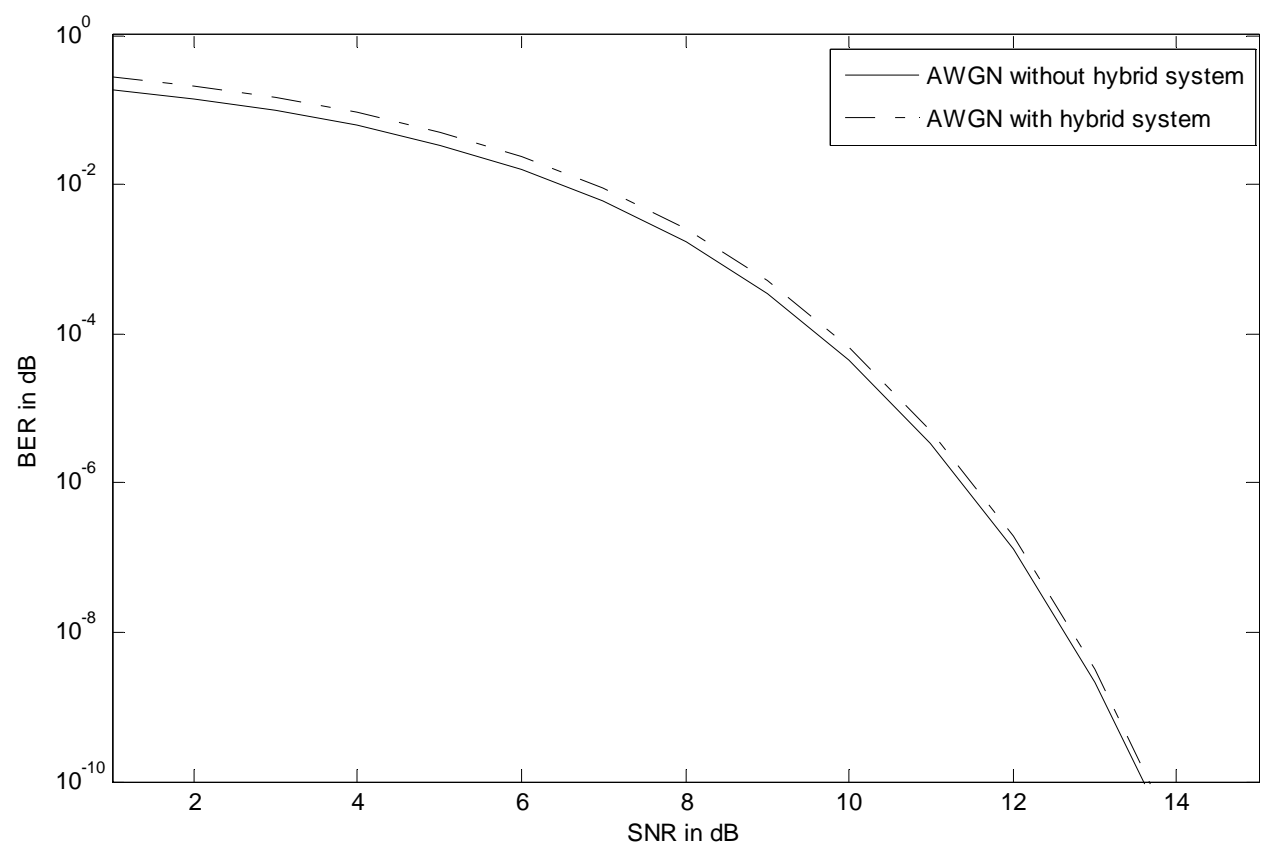

Fig. 3 Comparison between BER of AWGN channel with and without hybrid DS/SFH 
Proceedings of the $5^{\text {th }}$ ICEENG Conference, 16-18 May, 2006

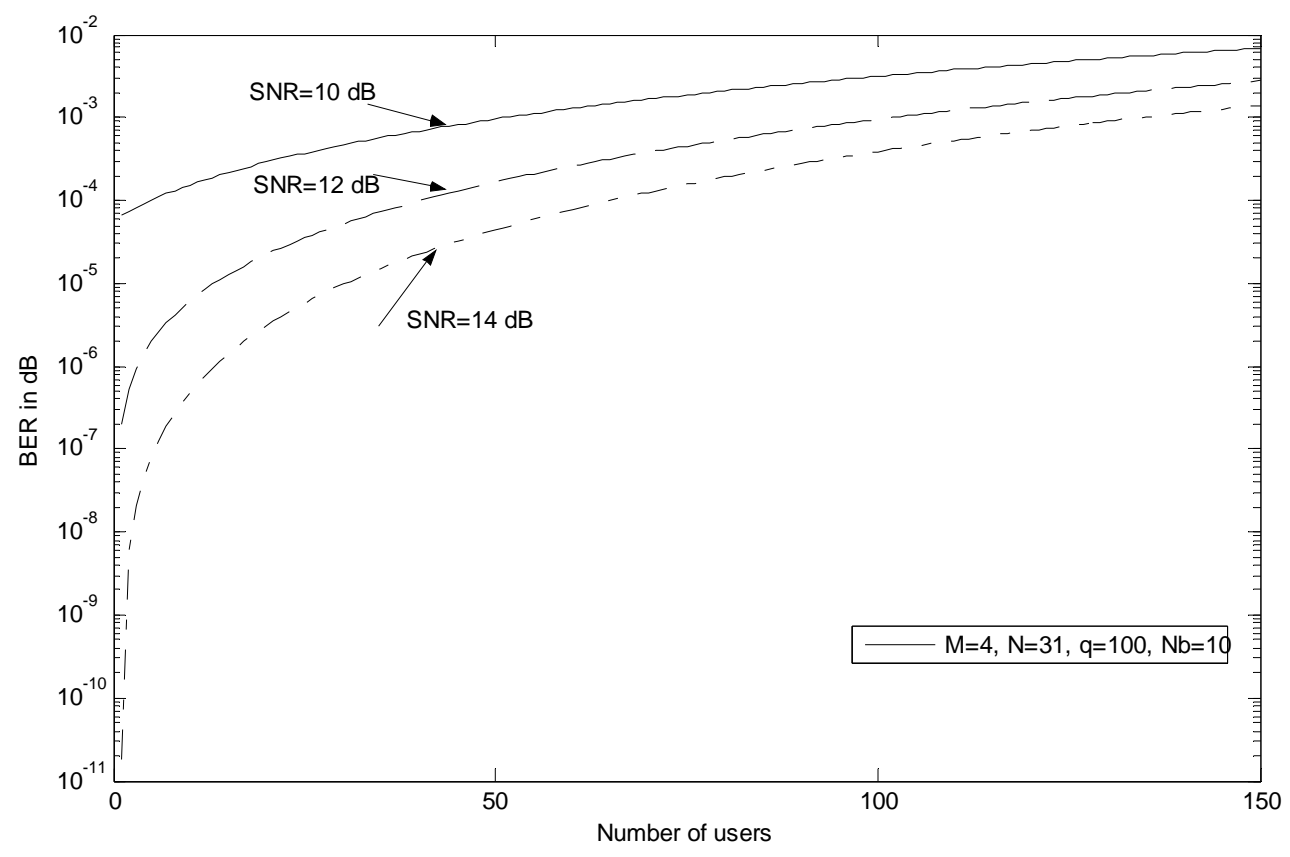

Fig. 4 System capacity of the hybrid DS/SFH over AWGN with different values of SNR

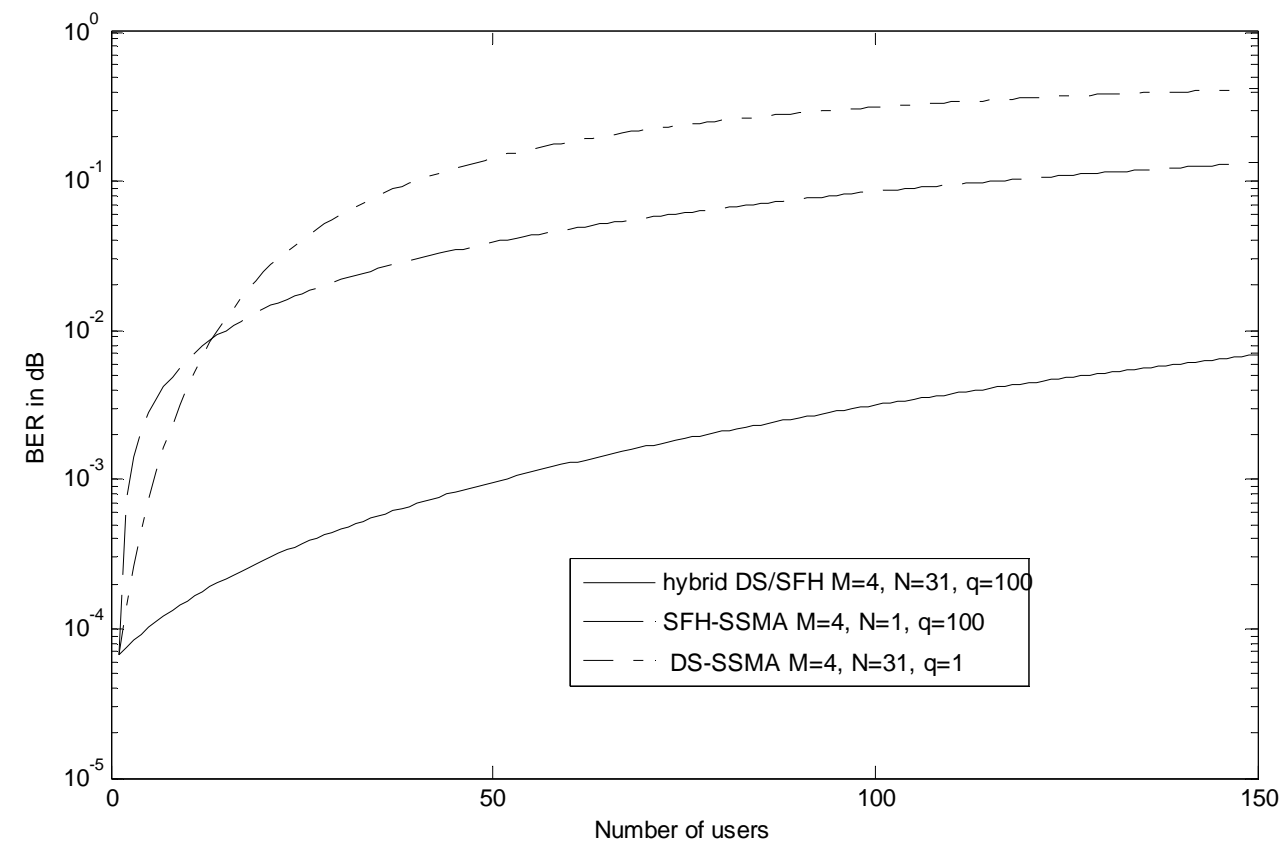

Fig. 5 Comparison between system capacity of the hybrid Ds/SFH, FH, and DS/SSMA 